\title{
Conventional grade 1 chondrosarcoma: a challenging diagnosis with important implications on therapy and prognosis
}

\author{
Gabriel Veniamin Cozma1), Laurentiu Vasile Sima1), Raluca Maria CloşCA ${ }^{2)}$, Flavia Baderca ${ }^{2,3)}$,

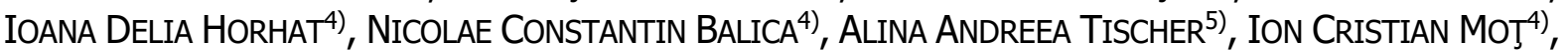 \\ Daniel Claudiu Malitja ${ }^{6}$, Aurel Marin ${ }^{7}$ ), CRistian ANDrei SARĂU ${ }^{8)}$
}

\author{
1) Department of Surgical Semiology, Victor Babeş University of Medicine and Pharmacy, Timişoara, Romania \\ 2) Service of Pathology, Emergency City Hospital, Timişoara, Romania \\ ${ }^{3)}$ Department of Microscopic Morphology, Victor Babeş University of Medicine and Pharmacy, Timişoara, Romania \\ 4) Department of ENT, Victor Babeş University of Medicine and Pharmacy, Timişoara, Romania \\ ${ }^{5)}$ Department of ENT, Emergency City Hospital, Timişoara, Romania \\ 6) Department of Radiology and Medical Imaging, Victor Babeş University of Medicine and Pharmacy, Timişoara, \\ Romania \\ 7)PhD Student, Department of ENT, Victor Babeş University of Medicine and Pharmacy, Timişoara, Romania \\ 8) Department of Medical Semiology I, Victor Babeş University of Medicine and Pharmacy, Timişoara, Romania
}

\begin{abstract}
Chondrosarcoma (CHS) is a malignant tumor of soft tissue with cartilaginous differentiation that represent one tenth of all malignant proliferations developed from bone tissues. Even if CHS represents the third malignancy with bone localization, after myeloma and osteosarcoma, it is far less diagnosed in the head and neck region. The current paper presented two cases of conventional CHSs, which were diagnosed in Department of Thoracic Surgery and Department of Otorhinolaryngology of Emergency City Hospital, Timişoara, Romania, between February and June 2021. The malignant cases were of peripheral CHSs, one of scapula, and the other one had an extremely rare tracheal location with microscopic features of conventional low-grade tumors (grade 1). In all cases, conservative surgical curative treatment was performed, with a favorable postoperative evolution.
\end{abstract}

Keywords: chondrosarcoma, immunohistochemistry, tracheal malignancy, atypical chondrocytes.

\section{a Introduction}

Chondrosarcomas (CHSs) are uncommon diagnosed malignancies characterized by cartilage matrix synthesis. The annual incidence has been reported as one to 200000 [1]. CHSs can develop in contact with an osseous structure formed by endochondral osteogenesis. The proximal bone of both extremities and the pelvis are by far, the most common affected. The second place of occurrence as incidence is represented by the bones of thorax, skull, and tibia. The tumors arise from the hyaline cartilage that cover the bone's epiphysis. The lesions can appear de novo or as a malignant transformation of a benign tumor as chondroma, exostoses, Ollier disease and Maffucci syndrome. The incidence of CHSs in the head and neck region is variable, the sites most affected being the jaws and vertebrae of cervical region. CHSs can also be diagnosed in various extra-osseous sites, in the soft tissue nearby a bone or, rarely, in organs that contain cartilage in their structure as trachea or larynx [2-10]. Tracheal and laryngeal cartilaginous neoplasms occur in hyaline cartilage and only very rare in elastic cartilage, and they are extremely rare [11-13].
There are two main subtypes of CHS: conventional and non-conventional. Conventional CHS accounts for 85$90 \%$ of all cases and is subdivided into central, peripheral, and periosteal subgroups. Non-conventional CHSs include three types: mesenchymal, clear-cell and dedifferentiated CHSs [1].

CHSs present in patients between 40 years and 60 years [14].

The symptoms of CHSs developed in a preexisting bone are non-specific and include swelling and local pain for a long period of time, not assigned at anti-inflammatory drugs. Dyspnea, wheeze, cough, hemoptysis, hoarseness is present in tracheal location [15-17]. CHS usually presents as a solitary lesion.

Grossly, CHSs are firm tumors with lobulated appearance, with slippery, glassy, grayish cut surface.

The microscopic exam reveals slightly pleomorphic chondrocytes embedded in a cartilaginous matrix. The myxoid change, calcification or necrosis can sometimes occur. Usually, the atypical chondrocytes present dark, hyperchromatic nuclei. The most important in the treatment of CHS and its prognostic factors is the grading system pronounced on histological criteria $[18,19]$. The features

This is an open-access article distributed under the terms of a Creative Commons Attribution-NonCommercial-ShareAlike 4.0 International Public License, which permits unrestricted use, adaptation, distribution and reproduction in any medium, non-commercially, provided the new creations are licensed under identical terms as the original work and the original work is properly cited. 
useful for grading are cellularity, pleomorphism, multinucleation, and mitoses. The most common are the grade 1 tumors (low-grade sarcoma), with a favorable prognosis and a slowly progressive growth, although the local recurrence rate is relatively high $[20,21]$. In general, immunohistochemical (IHC) reactions play a limited role in the diagnosis of CHSs, which relies on morphological, clinical, and radiological features. The only consistent IHC finding is S100 protein positivity.

CHSs are resistant to chemotherapy and radiation therapy. The large resection of the tumor with free margins increases the 5 -year survival rates to $78 \%$. The treatment of choice for tracheal and laryngeal CHSs is conservative surgery, function-preserving surgery, including laser therapy [18-21].

\section{Aim}

The aim of the present study was to highlight the importance of the prompt diagnosis of grade 1 CHS in incipient stage, based on clinical features, radiologically aspects, and histopathological (HP) descriptions, bearing in mind that this histological grade has a favorable evolution.

\section{$\square$ Patients, Materials and Methods}

The current paper presented two cases of conventional CHSs with rare localization, one scapular and one tracheal, diagnosed in the Emergency City Hospital of Timişoara, Romania, in February 2021 and the second, in June 2021.

All the data regarding personal and familial medical history, and laboratory test results were noted.

To obtain the diagnosis, the lesions were biopsied and sent to the Service of Pathology of the Hospital in 10\% $(v / w)$ neutral buffered formalin, where the harvested specimens were embedded in paraffin. Serial sections, $4 \mu \mathrm{m}$-thick, displayed on silanized slides were prepared for the diagnosis using morphological Hematoxylin-Eosin (HE) staining. To complete the diagnosis, histochemical Periodic AcidSchiff(PAS) reaction for mucin and additional IHC reactions were used. All the data regarding the antibodies used for IHC reactions are centralized in Table 1 . All the antibodies and the reagents utilized for immunohistochemistry were acquired from Novocastra ${ }^{\mathrm{TM}}$, Leica Biosystems.

Both patients approved their participation in the study by signing the informed consent of Ethics Committee from both, Hospital and University.

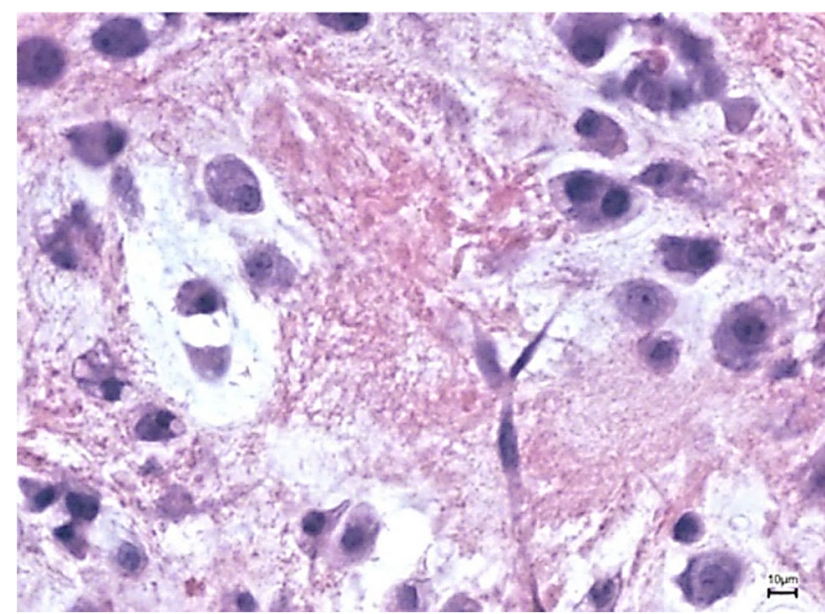

Figure 1 - Binucleated chondrocytes in cartilaginous matrix. HE staining, $\times 400$. HE: Hematoxylin-Eosin.
Table 1 - Data related to the antibodies used for immunohistochemical reactions

\begin{tabular}{cccc}
\hline Antibody & Substrate & Clone & Dilution \\
\hline Ki67 & Monoclonal mouse & MM1 & $1: 200$ \\
\hline S100 protein & Polyclonal rabbit & EP32 & $1: 100$ \\
\hline p53 & Polyclonal mouse & CM5 & $1: 200$ \\
\hline E-cadherin & Monoclonal mouse & 36B5 & $1: 25$ \\
\hline
\end{tabular}

\section{Case presentations}

\section{Case No. 1}

A 49-year-old female patient was hospitalized in February 2021 with arthralgia in the left shoulder. Personal medical history was insignificant for present lesion, also the medical treatment. She did not consume alcohol and was non-smoker. At clinical examination, no other abnormalities or palpable lymphadenopathy besides scapular lesion were identified. Paraclinical tests showed: erythrocyte sedimentation rate $70 \mathrm{~mm} / \mathrm{h}$, hemoglobin $14 \mathrm{~g} / \mathrm{dL}$, hematocrit $41.2 \%$, serum creatinine $0.55 \mathrm{mg} / \mathrm{dL}$. Chest radiography showed normal images. The computed tomography scan revealed peripheral scapular tumor of $4 / 3.4 \mathrm{~cm}$ with muscle and bone invasion.

Under general anesthesia, a suprascapular incision with infraspinous muscle and teres major excision and partial resection of scapular body was performed. The postoperative evolution was favorable.

Macroscopically, on the cut surface, the lesion was characteristically glassy, lobulated, and firm.

The HP examination of HE-stained slides revealed a malignant partially encapsulated proliferation of atypical chondrocytes in cartilaginous matrix, showing a lobulated pattern of growth. The tumor cells presented basophilic or pale cytoplasm, with hyperchromatic nuclei, and minimally pleomorphism. Some of the cells were binucleated (Figure 1). PAS reaction highlighted intracytoplasmic positive inclusion (Figure 2). The chondroid cells were heterogeneously positive for S100 protein, with intense positive areas merged with negative zones and negative for p53 protein and E-cadherin (Figures 3-8). The Ki67 index was high (80\%) at tumor invasion front and low in other areas (Figures 9 and 10). Lobules of atypical chondrocytes invaded the striated muscle, adipose and fibrous connective tissues, and osseous structures of scapula. The final diagnosis of peripheral low-grade CHS with bone invasion and negative margins was signed out.

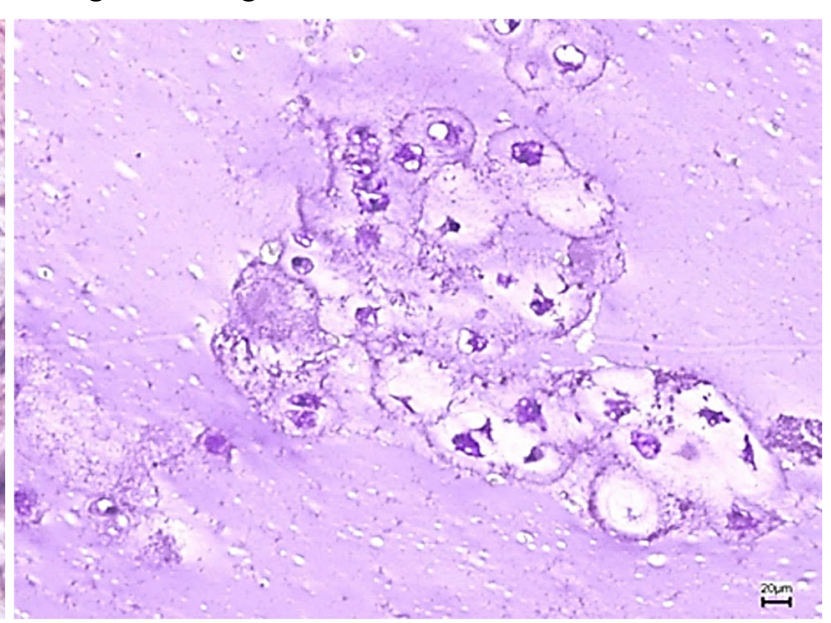

Figure 2 - Atypical chondrocytes with intracytoplasmic inclusions. PAS reaction, $\times 200$. PAS: Periodic Acid-Schiff. 


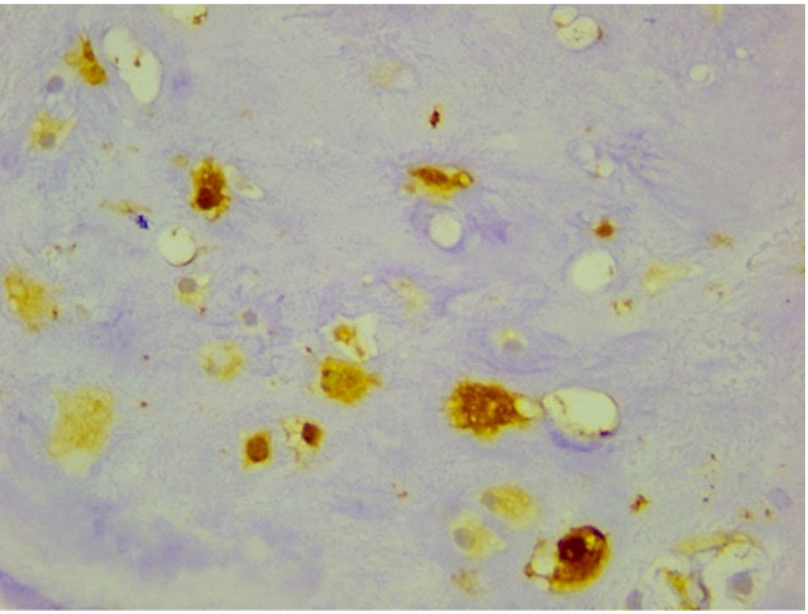

Figure 3 - Most of the tumor chondrocytes were immunohistochemically positive for $\mathbf{S 1 0 0}$ protein, $\times 200$.

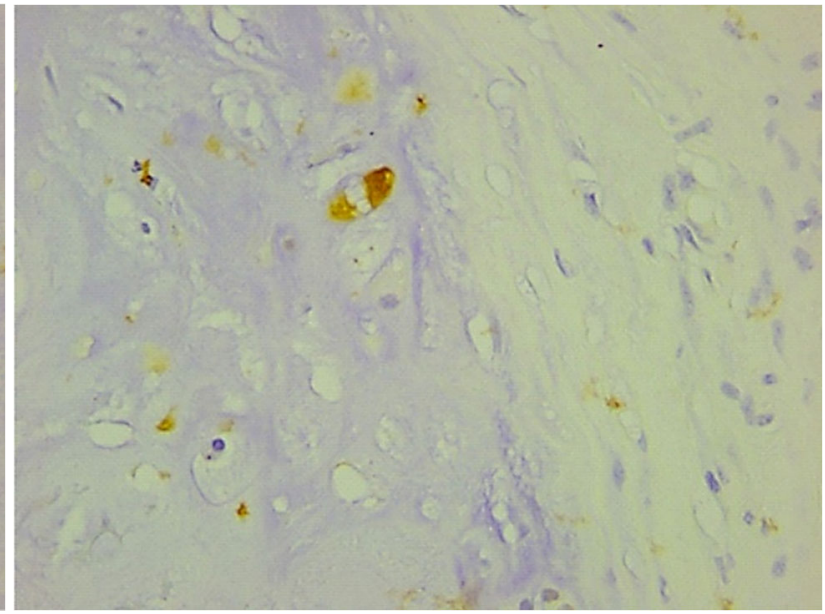

Figure 4 - Atypical chondroblast in mitosis, immunohistochemically positive for $S 100$ protein, $\times 100$.

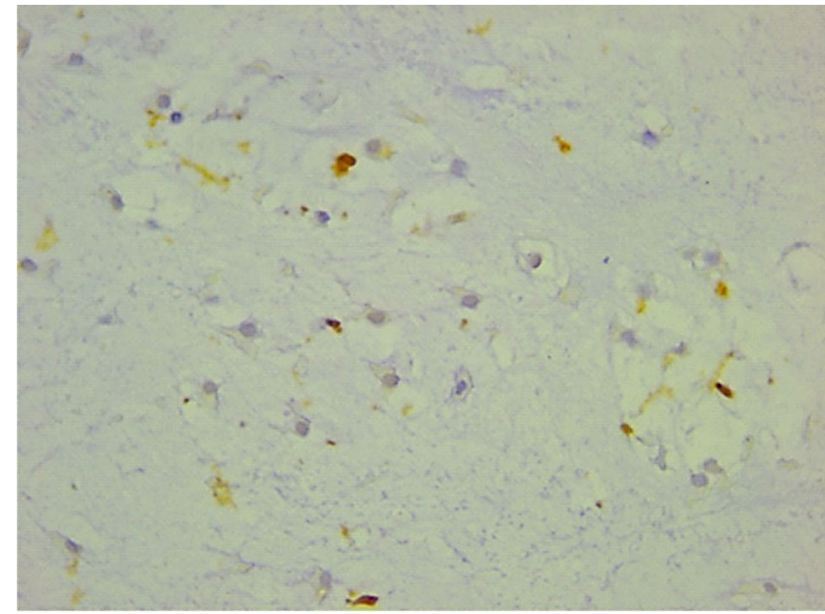

Figure 5 - Tumor area showing negative immunohistochemical reaction for $S 100$ protein, $\times 100$.

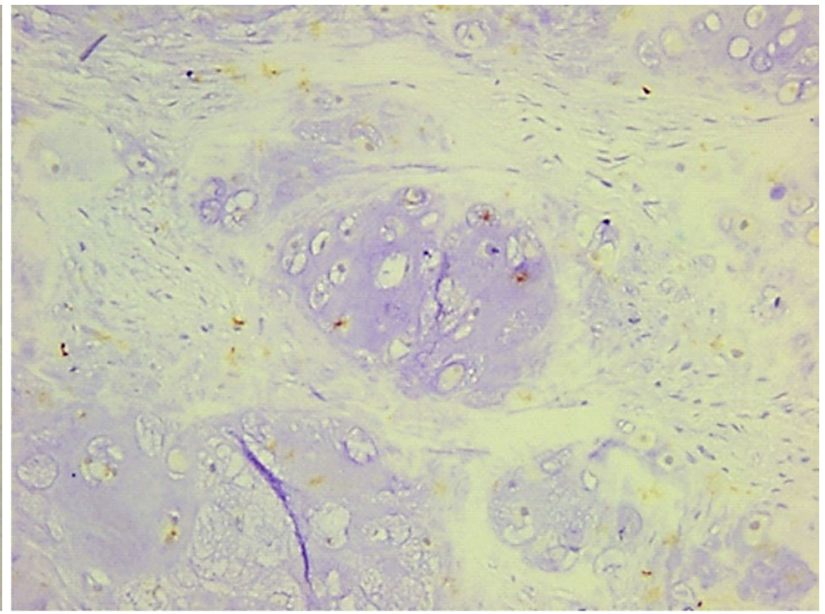

Figure 6 - Lobules of malignant chondrocytes, with negative immunohistochemical reaction for $\mathbf{S 1 0 0}$ protein, $\times 50$.

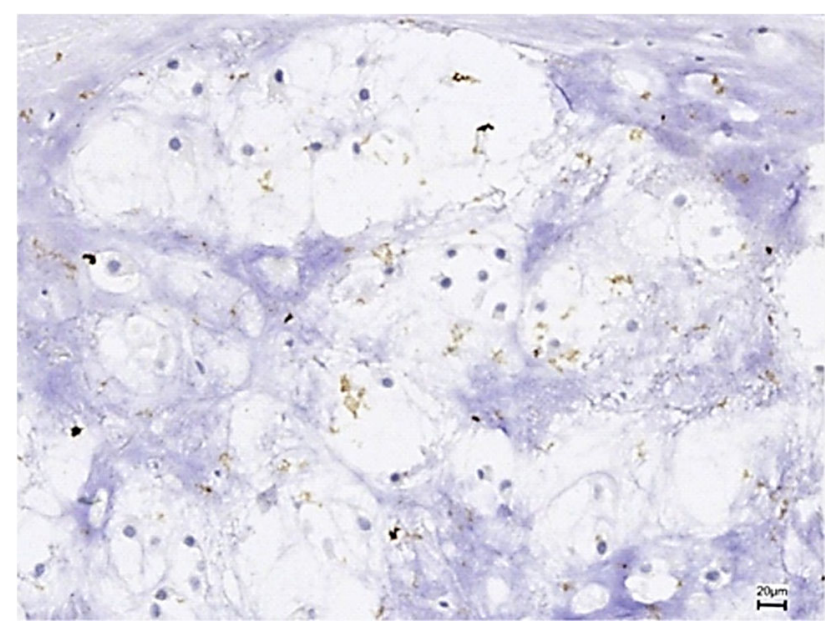

Figure 7 - Tumor area showing negative immunohistochemical reaction for E-cadherin, $\times 200$.

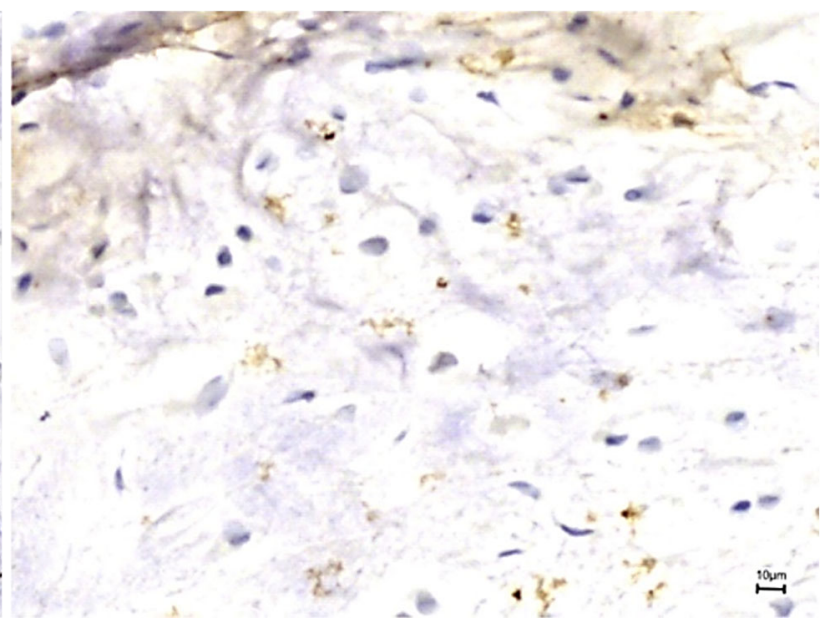

Figure 8 - Lobules of malignant chondrocytes, with negative immunohistochemical reaction for p53, $\times 400$. 


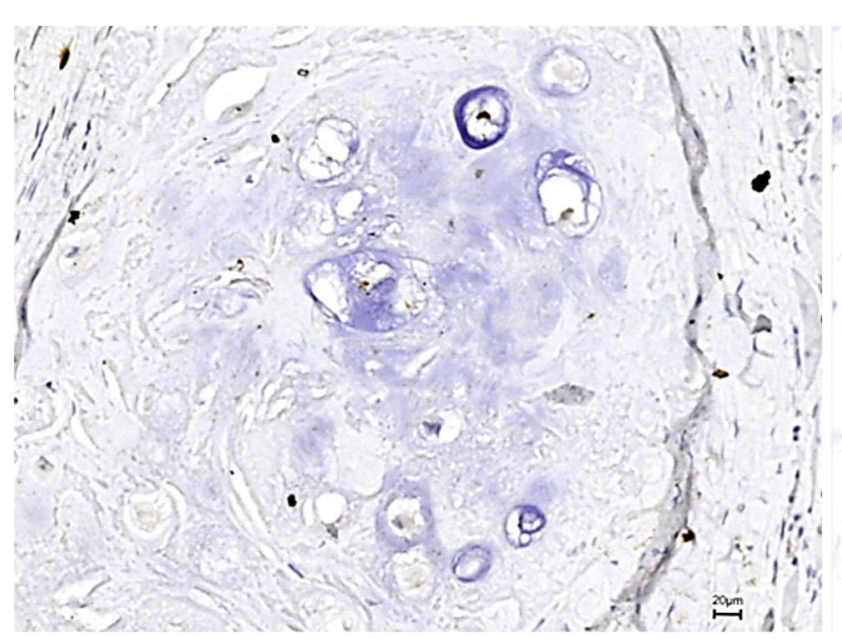

Figure 9 - Tumor area showing negative immunohistochemical reaction for Ki67, ×400.

After the surgical intervention, the patient was discharged without complications and is currently being monitored by her oncologist.

\section{Case No. 2}

The patient, a 64-year-old female, presented in June 2021 for dyspnea, cough, and hoarseness for about one month with repeated episodes of wheeze and hemoptysis about one week.

On personal medical background was noted hypertension treated with beta-blockers and family history of cardiac diseases. The patient was non-smoker and did not consume alcohol.

Laryngoscopy documented the existence of a proliferated region at subglottic level, $1 / 1.5 \mathrm{~cm}$ in size, covered by smooth mucosa, with endoluminal occlusion that occupied about $70 \%$ of the lumen. Chest radiography reveals a lytic tracheal lesion, with dense spicules of calcification and an eccentric, lobular appearance. Pulmonary normal X-ray image.

Under general anesthesia, emergency tracheostomy and suspended microlaryngoscopy was performed. The surgical treatment was conservative with laser $\mathrm{CO}_{2}$ therapy and function-preserving surgery. After two days, tracheal decannulation was practiced, with anterior cervical suture after another three days. A new exploratory laryngoscopy

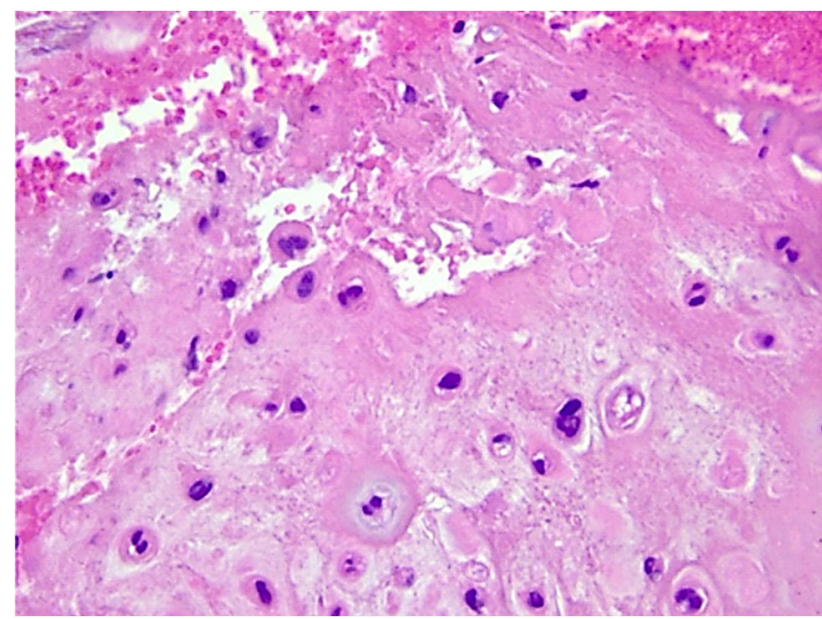

Figure 11 - Atypical chondrocytes, some of them binucleated. HE staining, $\times 400$.

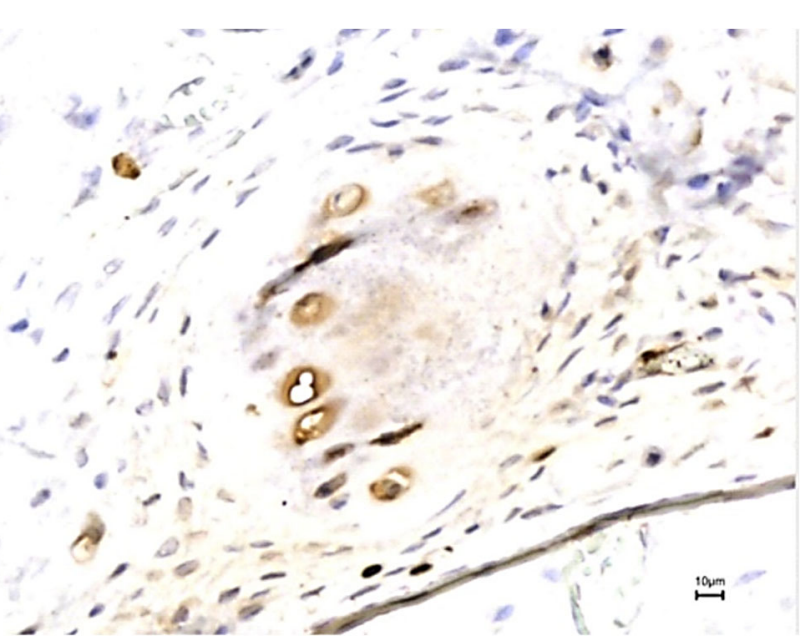

Figure 10 - Lobules of malignant chondrocytes, with positive immunohistochemical reaction for Ki67, ×200.

was performed, and the tracheal space was free.

Grossly, the biopsy fragments presented a lobulated, glassy, firm, and white or gray cut surface. The HP examination of HE-stained slides revealed a malignant tumor composed of the hyaline cartilaginous resembling proliferated lobules, that invaded the perichondrium (Figure 11). The tumor was situated in lamina propria of a mucosa nearby foci of elastic cartilage and disorganizing the seromucous acini (Figure 12). The lobules of tumor chondrocytes disrupted the respiratory columnar ciliated pseudostratified epithelium or area of mature squamous metaplasia (Figures 13 and 14). The tumor consisted of atypical chondrocytes, with large quantity of basophilic cytoplasm, and enlarged nuclei with big nucleoli, some of them showing highly nuclear pleomorphism and acidophilic inclusions in cytoplasm (Figure 15). An important number of atypical chondrocytes were binucleated (Figure 16). PAS reaction highlighted the presence of cytoplasmic inclusions (Figures 17 and 18). The tumor destroyed the ossified hyaline cartilage and presented foci of osseous metaplasia (Figures 19 and 20). The tumor cells were heterogeneously positive for $\mathrm{S} 100$ protein, and negative for $\mathrm{p} 53$ protein and E-cadherin (Figures 21-23). The Ki67 index was 10\% (Figure 24).

The diagnosis of conventional grade 1 CHS affecting the proximal part of the trachea was pronounced and the patient was referred for oncological treatment.

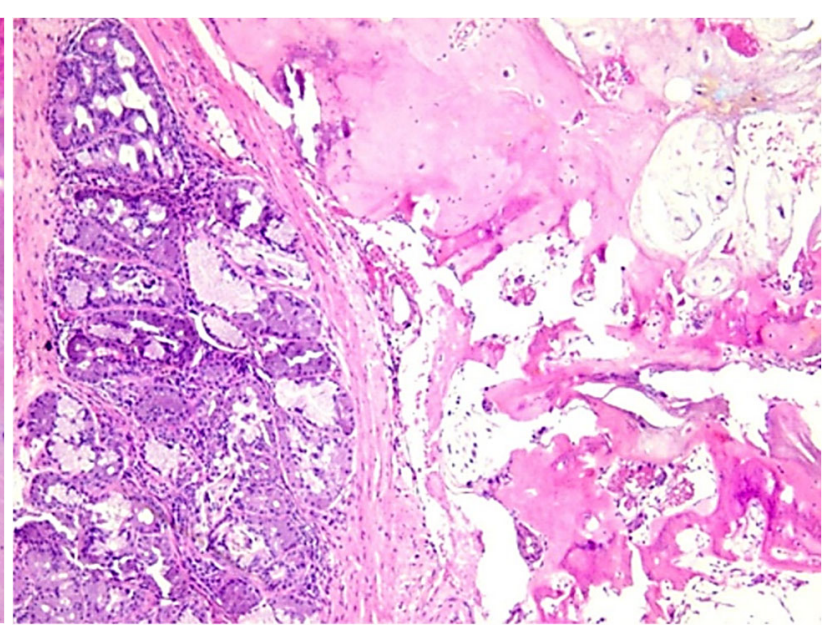

Figure 12 - The tumor infiltrated the seromucous acini. HE staining, $\times 100$. 


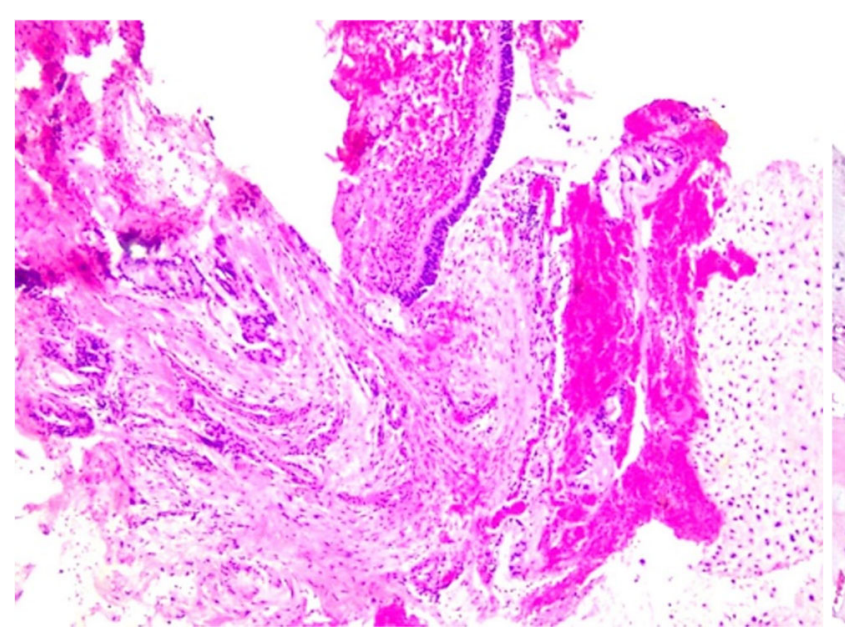

Figure 13 - Respiratory mucosa lined by pseudostratified columnar epithelium disorganized by the tumor. $\mathrm{HE}$ staining, $\times 50$.

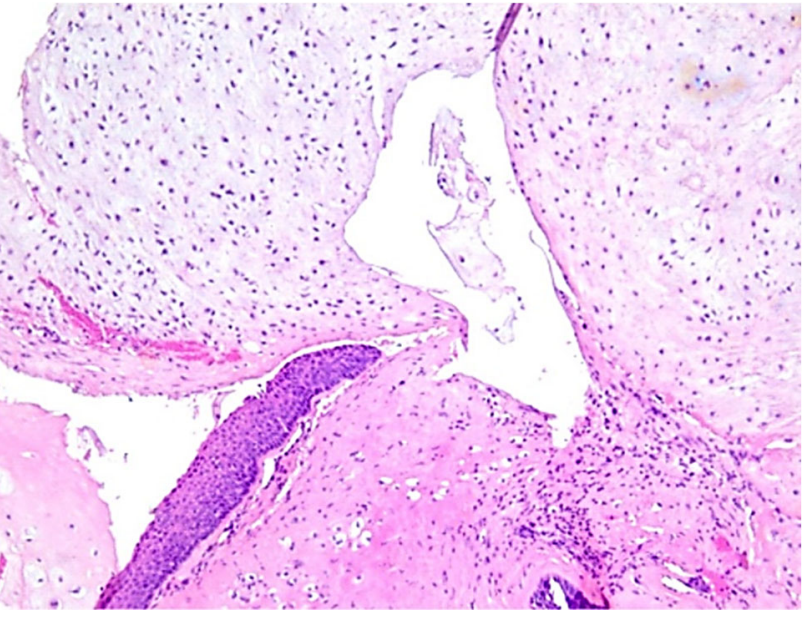

Figure 14 - Area of mature squamous metaplasia disrupted by the tumor. HE staining, $\times 100$.

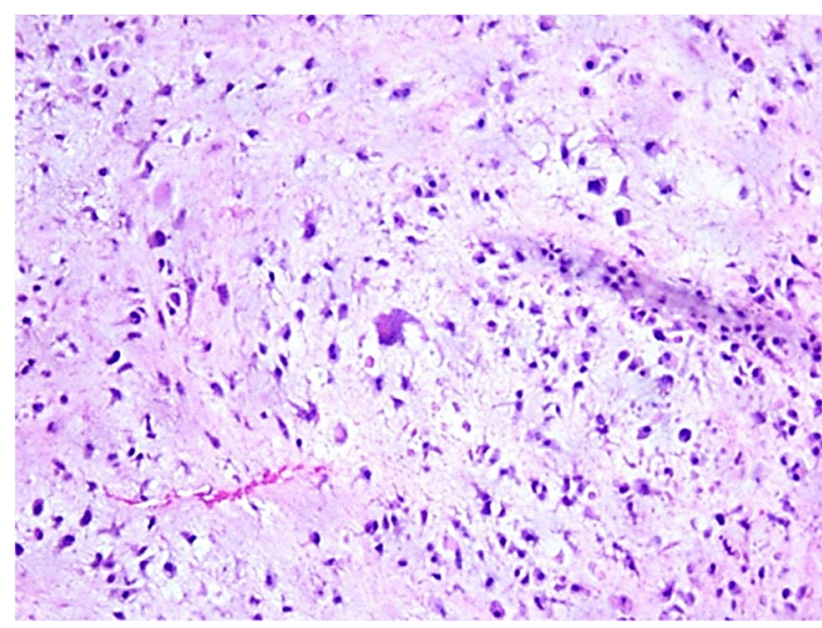

Figure 15 - Atypical chondrocytes with intracytoplasmic inclusions, in a myxoid stroma. HE staining, $\times 200$.

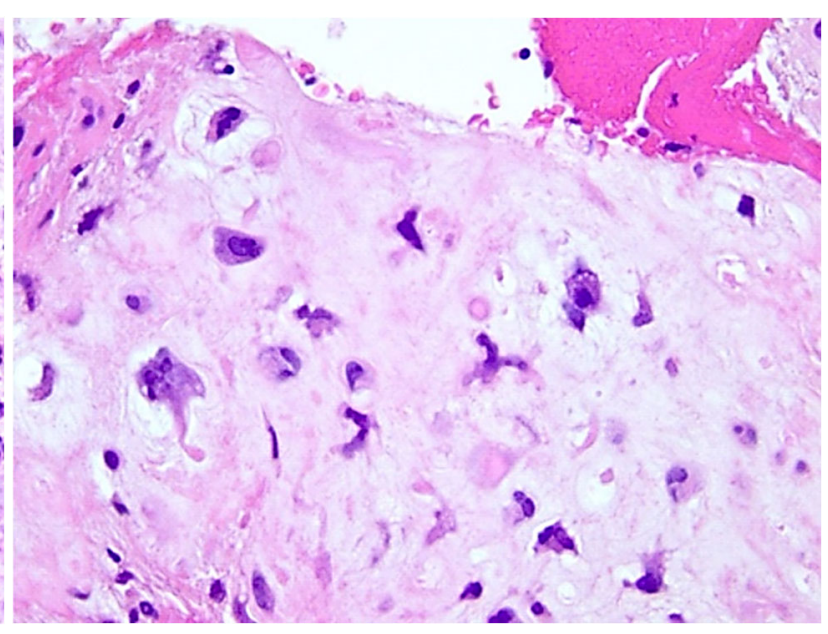

Figure 16 - Atypical chondrocytes with intracytoplasmic inclusions. HE staining, $\times 400$.

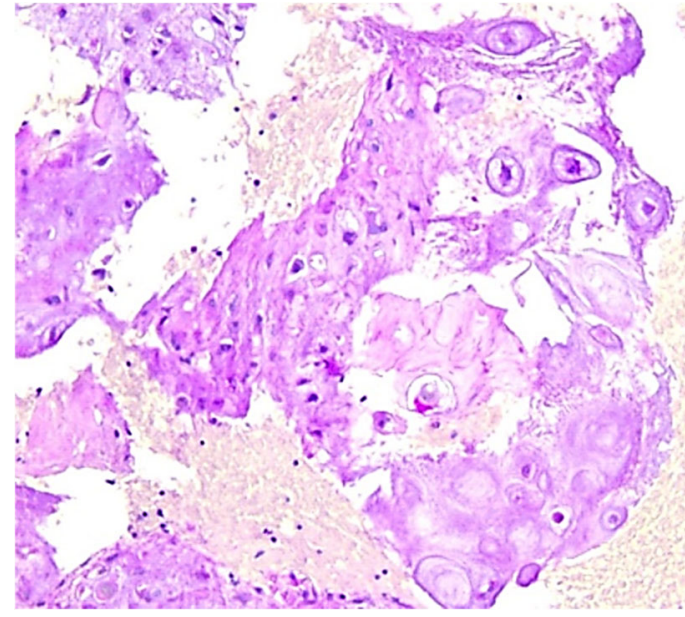

Figure 17 - Atypical chondrocytes with intracytoplasmic inclusions. PAS reaction, $\times 200$.
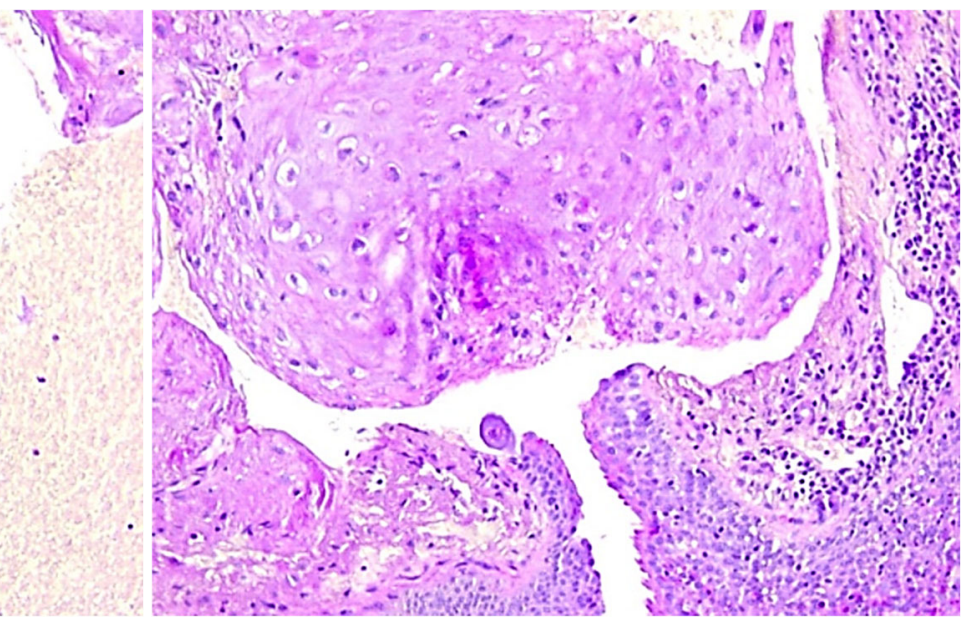

Figure 18 - Atypical chondrocytes with intracytoplasmic inclusions, invading the lining epithelium. PAS reaction, $\times 200$. 


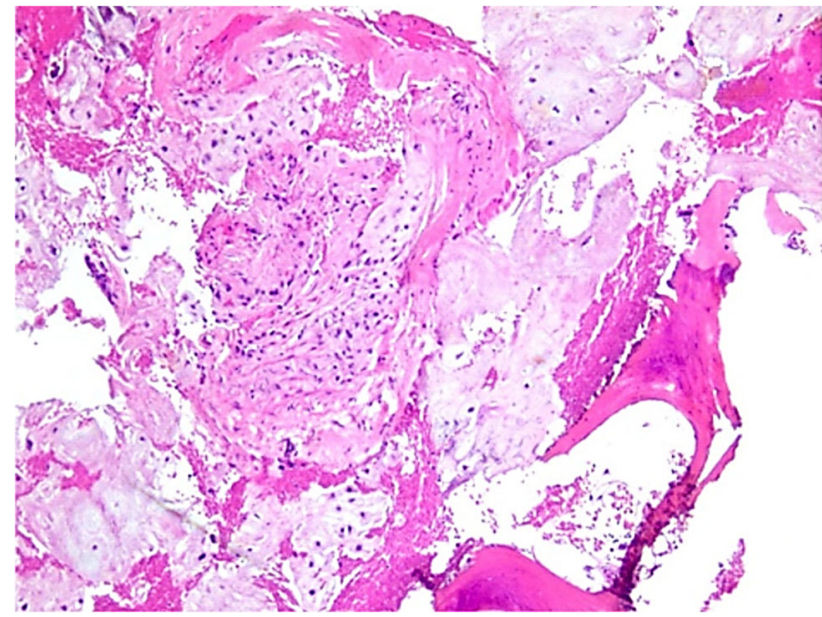

Figure 19 - Atypical chondrocytes invaded ossified cartilage. HE staining, $\times 100$.

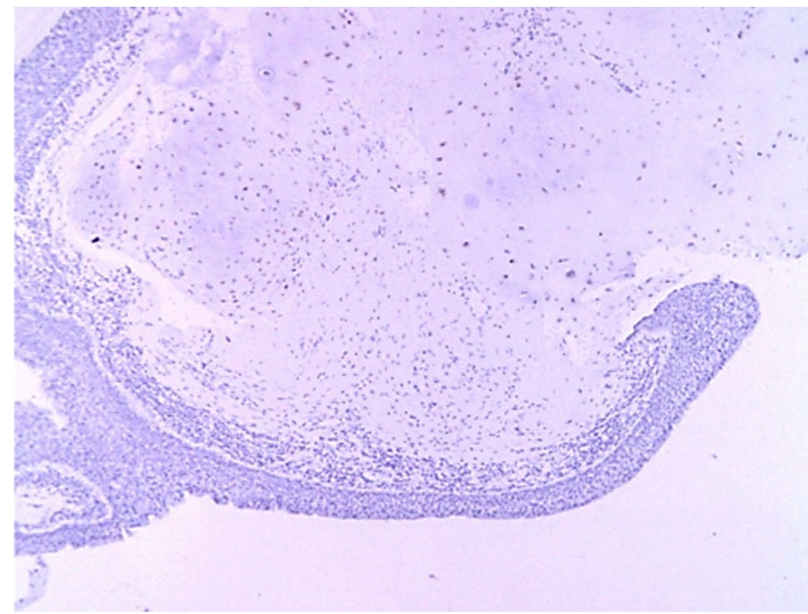

Figure 21 - Atypical chondrocytes immunohistochemically positive for $S 100$ protein, $\times 50$.

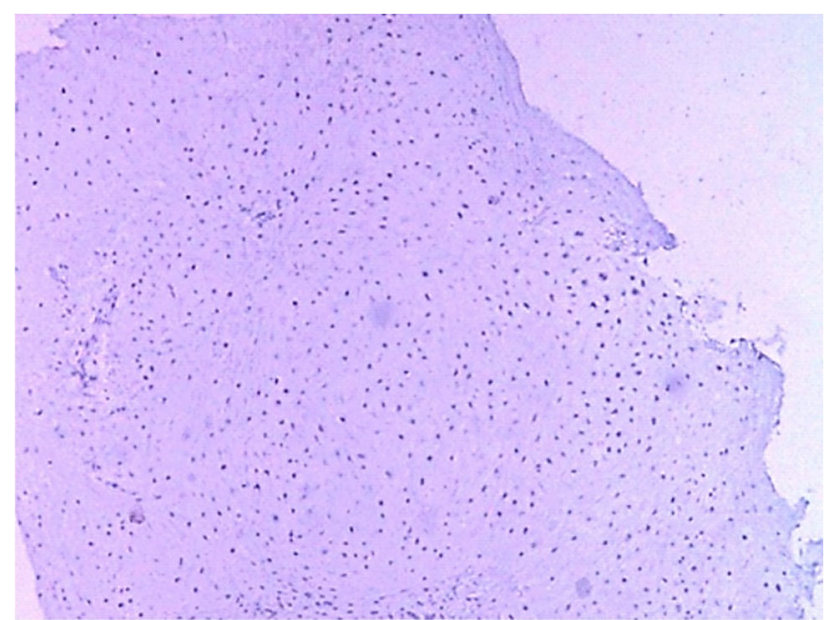

Figure 23 - The negative immunohistochemical reaction for E-cadherin, $\times 50$.

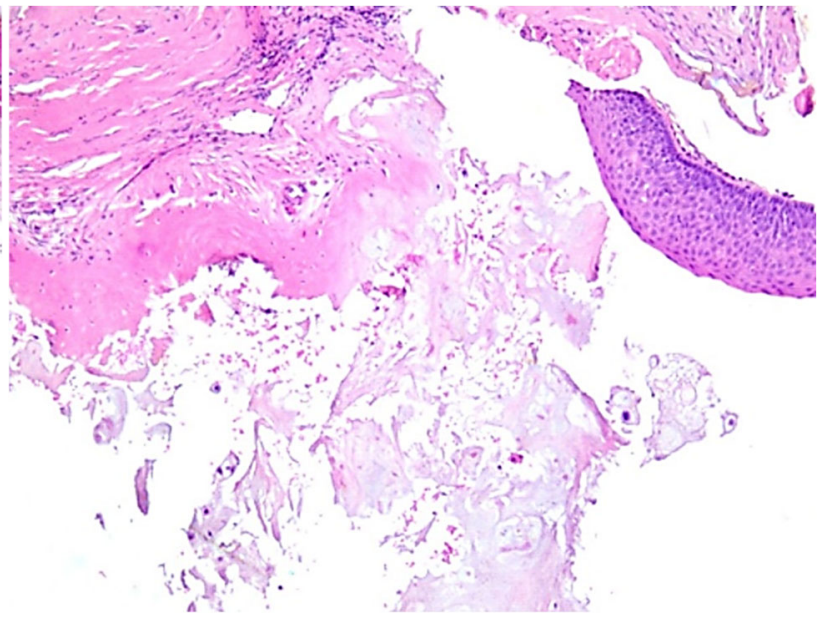

Figure 20 - The tumor showed areas of osseous metaplasia. HE staining, $\times 100$.

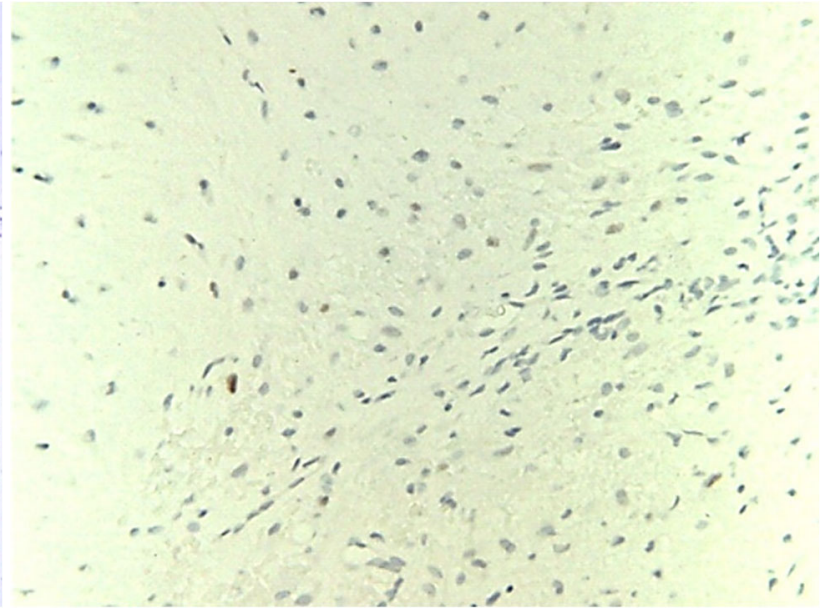

Figure 22 - The negative immunohistochemical reaction for $p 53, \times 200$.

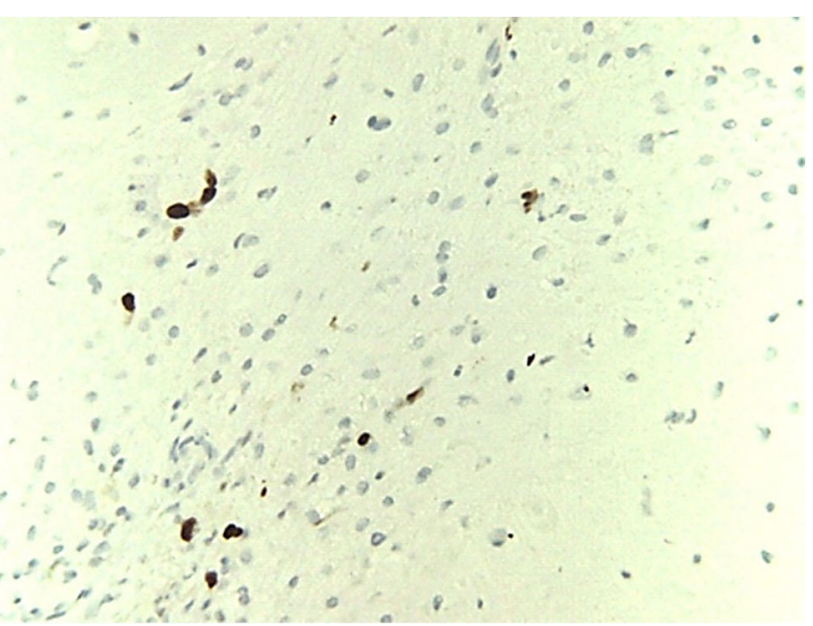

Figure $24-$ Ki67 index in atypical cells, $\times 200$. 


\section{口 Discussions}

The CHSs are malignant mesenchymal bone tumors composed of atypical chondrocytes embedded in chondroid matrix. After osteosarcomas and myelomas, CHSs rank third in the group of primary malignant bone tumors [22, 23].

The etiology of CHSs remains unclear, although several hypotheses have been proposed. Disordered ossification found in hyaline cartilage in older patients, occurs in areas of muscle insertions, and may serve as a nidus for tumor development. Other predisposing factors are radiotherapy, injection of radioactive material, usually after a latent period of many years, or repeated trauma. In our cases, no predisposing or risk factors for CHSs were identified [24].

Histological features include a lobulated growth pattern, with disorganized chondrocytes. Lacuna occupied by doublepacked atypical chondrocytes are often seen. Tumor cells present mild pleomorphism, but the presence of nucleoli is a constant encountered feature. In conventional CHS, the histological grading represents the main prognostic factor. The features useful in tumor grading are cellularity, pleomorphism, multinucleation and mitoses. Most CHSs are grade 1 (low grade), with a very good prognostic. They show a pattern of lobular disarray and destructive invasion of native cartilage and bone. Moderately differentiated tumors (grade 2) show a higher degree of cellularity and nuclear pleomorphism than grade 1 tumors and may have few mitoses. Grade 3 tumors have high cellularity, significant multinucleation, nuclear atypia, hyperchromic nuclei, areas of necrosis, and increased mitotic index [1].

In the present study, there were performed IHC markers (S100 protein, Ki67 index, p53, E-cadherin), and histochemical stainings both for diagnostic and outcome purposes. According to the published data, the Ki67 index can be used in the diagnosis and prognosis of cartilaginous tumors, malignant cartilaginous tumors having a greater $\mathrm{Ki} 67$ index in comparison to the benign counterparts $[25,26]$. The scapular tumor presented a high Ki67 index (80\%), helping us to rule out an enchondroma. A study on cartilaginous tumors shows that the Ki67 index has a higher immunoreactivity $(p=0.0044)$ in comparison to enchondromas, recommending using it as a marker in the diagnosis of cartilaginous tumors [25]. Another study on CHSs indicates the value of Ki67 index as a prognostic factor for this type of tumors, suggesting that patients with a high Ki67 index might benefit from adjuvant therapy, immunotherapy, or biological modulation [26]. Taking into consideration that the scapular tumor has a higher Ki67 index (80\%) than the tracheal tumor $(10 \%)$, we conclude that the scapular tumor could benefit from adjuvant or immunomodulatory therapy. The same prior mentioned study shows the existence of PAS-positive intracytoplasmic globules, suggesting that hyaline intracytoplasmic globules were more frequently found in malignant than in benign cartilaginous neoplasms ( $p=0.042)$ [25]. Relying on this finding, we performed a PAS staining, which highlighted the presence of these intracytoplasmic granules, thus helping in the differential diagnosis between malignant and benign.

There are few studies in the literature regarding p53 and E-cadherin immunoreactivity in high-grade CHSs. Nevertheless, a study performed on 39 CHSs shows that
17 of them are immunoreactive for p53 and concludes that a direct correlation between this expression and the outcome in CHSs could not be established, which is also our fact that we also sustain [26].

Microscopically, our cases were conventional low-grade CHSs, which coincides with the data in the literature. Based on histological and imagistic aspects, CHS of the scapula was considered peripheral, with bone invasion.

Tracheal CHSs are rarely reported. With age, hyaline cartilage from different parts of the body undergoes ossification. It is presumed that during this process pluripotential mesenchymal cells reach the cartilage and could be the source of malignant cartilaginous tumors. The ossification of hyaline cartilage is intensified with age, the frequency of the tumor is higher between fifth and eighth decades of life corresponding to the high rates of bone formation.

Tracheal CHSs have been a diagnostic challenge for both clinicians and pathologists. The differential diagnosis includes chondroma, fracture callus and tracheopathia chondroplastica. The last is a condition restricted to trachea, with unidentified etiology. Laryngoscopy reveal gritty small submucosal nodules. The tumor interests the part of trachea that includes hyaline cartilage, the posterior wall not being involved. Microscopically, the connective tissue of lamina propria presents foci of microcalcification and area of newly formed bone. Clinical and imagistic correlation can aid in this distinction. The fractures can interest the ossified hyaline cartilage of trachea. The resulting callus may represent an important differential for $\mathrm{CHSs}$, but the callus is not lobulated and do not show pushing margins as malignant tumor does, even if the osseous trabeculae are lined by osteoblasts. Usually, the clinicians can point out a previous trauma. For tracheal tumors, the differential diagnosis, before surgery, between a benign cartilaginous tumor and its malignant counterpart cannot be done. For the CHSs arisen in the long bones, the differential diagnosis with a benign tumor imposes the surgical procedure. The decision is a teamwork between clinicians and radiologists. On X-ray exam, benign cartilaginous tumors of long bones are encapsulated and do not destroy bone cortex. Because of the architectural particularities of tracheal ossified cartilage with restricted medullary space, even a benign tumor can destroy the bone cortex. For that, the preoperative distinction between chondromas and CHSs is moot. On histological grounds, even if, similar to malignant cartilaginous tumor, chondromas present hypercellular areas and an intensification of matrix eosinophilia, the tumor cells show no atypia and the nuclear cytoplasmatic ratio is unchanged.

Grade 1 conventional-type CHS is a slow-growing neoplasm and is resistant to chemotherapy and radiation therapy $[16,27]$. Alternative therapeutical methods can be tested to enhance the treatments benefits and to improve the patient's life quality [28]. The tumorectomy of bones CHSs with free margins increase the life expectancy at five years from $54 \%$ to $78 \%$ [29].

The prognosis for tracheal CHS is favorable although the local recurrence rate is relatively high (18-50\%), usually due to incomplete resection [30]. Distant metastases are very rare. The treatment of choice is conservative surgery, function-preserving surgery, including laser therapy [31-33]. 


\section{$\square$ Conclusions}

CHSs are very rare mesenchymal tumors, and their diagnosis is a challenge for pathologists. Low-grade CHS requires a differential diagnosis with enchondroma and reactive changes. This was done by correlating the histological aspects in the usual staining, with the result of IHC and histochemical reactions, as well as with clinical and imaging data. The article presented two interesting cases of conventional grade $1 \mathrm{CHS}$ with different location, one of them with a very rare presentation.

\section{Conflict of interests}

The authors declare that they have no conflict of interests.

\section{Authors' contribution}

Gabriel Veniamin Cozma and Laurenţiu Vasile Sima equally contributed to the manuscript.

\section{References}

[1] Fletcher CDM, Unni KK, Mertens F (eds). Pathology and genetics of tumours of soft tissue and bone. $3^{\text {rd }}$ edition, vol. 5 , World health Organization (WHO) Classification of Tumours, International Agency for Research on Cancer (IARC) Press, Lyon, France, 2006, 130-132. https://publications.iarc.fr/BookAnd-Report-Series/Who-Classification-Of-Tumours/PathologyAnd-Genetics-Of-Tumours-Of-Soft-Tissue-And-Bone-2002

[2] van Praag Veroniek VM, Rueten-Budde AJ, Ho V, Dijkstra PDS; Study group Bone and Soft tissue tumours (WeBot); Fiocco M van de Sande MAJ. Incidence, outcomes and prognostic factors during 25 years of treatment of chondrosarcomas. Surg Oncol, 2018, 27(3):402-408. https://doi.org/10.1016/j.suronc.2018. 05.009 PMID: 30217294

[3] Kim MJ, Cho KJ, Ayala AG, Ro JY. Chondrosarcoma: with updates on molecular genetics. Sarcoma, 2011, 2011:405437. https://doi.org/10.1155/2011/405437 PMID: 21403832 PMCID: PMC3042668

[4] Mukherjee D, Chaichana KL, Gokaslan ZL, Aaronson O, Cheng JS, McGirt MJ. Survival of patients with malignant primary osseous spinal neoplasms: results from the Surveillance, Epidemiology, and End Results (SEER) database from 1973 to 2003. J Neurosurg Spine, 2011, 14(2):143-150. https:// doi.org/10.3171/2010.10.SPINE10189 PMID: 21184634

[5] Giuffrida AY, Burgueno JE, Koniaris LG, Gutierrez JC, Duncan R, Scully SP. Chondrosarcoma in the United States (1973 to 2003): an analysis of 2890 cases from the SEER database. J Bone Joint Surg, 2009, 91(5):1063-1072. https:// doi.org/10.2106/JBJS.H.00416 PMID: 19411454

[6] Thanigai S, Douraiswami B. Chondrosarcoma of metacarpal - a rare presentation. J Clin Orthop Trauma, 2013, 4(2):85-88. https://doi.org/10.1016/j.jcot.2012.10.002 PMID: 26403630 PMCID: PMC3880538

[7] Kutzner EA, Park JS, Zaheer S, Inman JC. Tracheal chondrosarcoma: systematic review of tumor characteristics, diagnosis, and treatment outcomes with case report. Case Rep Oncol Med, 2017, 2017:4524910. https://doi.org/10.1155/2017/452 4910 PMID: 28620556 PMCID: PMC5460443

[8] Mendonça V, Jorge M, Monteiro-Grillo I, Palhano MJ, Feijó S. Tracheal chondrosarcoma. Clin Transl Oncol, 2010, 12(8): 576-580. https://doi.org/10.1007/s12094-010-0557-x PMID: 20709656

[9] de Almeida JR, Pagedar NA, Keshavjee S, Gilbert R. Chondrosarcoma of the trachea in a patient with Maffucci syndrome. J Otolaryngol Head Neck Surg, 2010, 39(2):E12-E15. PMID: 20211090

[10] de Vincentiis M, Greco A, Fusconi M, Pagliuca G, Martellucci S Gallo A. Total cricoidectomy in the treatment of laryngeal chondrosarcomas. Laryngoscope, 2011, 121(11):2375-2380. https://doi.org/10.1002/lary.22337 PMID: 22020889

[11] Obeso S, Llorente JL, Díaz-Molina JP, Sánchez-Fernández R, Rodrigo JP, Suárez C. [Surgical treatment of head and neck chondrosarcomas]. Acta Otorrinolaringol Esp, 2010, 61(4): 262-271. https://doi.org/10.1016/j.otorri.2009.12.002 PMID: 20096816
[12] Wang $\mathrm{Q}$, Chen $\mathrm{H}$, Zhou S. Chondrosarcoma of the larynx: report of two cases and review of the literature. Int J Clin Exp Pathol, 2015, 8(2):2068-2073. PMID: 25973106 PMCID: PMC4396297

[13] Chin OY, Dubal PM, Sheikh AB, Unsal AA, Park RCW, Baredes S, Eloy JA. Laryngeal chondrosarcoma: a systematic review of 592 cases. Laryngoscope, 2017, 127(2):430-439. https://doi.org/10.1002/lary.26068 PMID: 27291822

[14] Oliveira JFDS, Branquinho FAPL, Monteiro ARRTN, Portugal MECC, Guimarães AMFS. Laryngeal chondrosarcoma - ten years of experience. Braz J Otorhinolaryngol, 2014, 80(4): 354-358. https://doi.org/10.1016/j.bjorl.2014.05.004 PMID: 25183187

[15] Buda I, Hod R, Feinmesser R, Shvero J. Chondrosarcoma of the larynx. Isr Med Assoc J, 2012, 14(11):681-684. PMID: 23240373

[16] Donati D, Colangeli S, Colangeli M, Di Bella C, Bertoni F. Surgical treatment of grade I central chondrosarcoma. Clin Orthop Relat Res, 2010, 468(2):581-589. https://doi.org/10. 1007/s11999-009-1056-7 PMID: 19727994 PMCID: PMC 2806970

[17] Gao CP, Liu JH, Hou F, Liu H, Xu WJ. Low-grade chondrosarcoma of the cricoid cartilage: a case report and review of the literature. Skeletal Radiol, 2017, 46(11):1597-1601. https:// doi.org/10.1007/s00256-017-2731-5 PMID: 28756568 PMCID: PMC5599471

[18] Fromm J, Klein A, Baur-Melnyk A, Knösel T, Lindner L, Birkenmaier C, Roeder F, Jansson V, Dürr HR. Survival and prognostic factors in conventional G1 chondrosarcoma. World J Surg Oncol, 2019, 17(1):155. https://doi.org/10.1186/s1295 7-019-1695-4 PMID: 31481076 PMCID: PMC6724259

[19] Bindiganavile S, Han I, Yun JY, Kim HS. Long-term outcome of chondrosarcoma: a single institutional experience. Cancer Res Treat, 2015, 47(4):897-903. https://doi.org/10.4143/crt. 2014.135 PMID: 25687868 PMCID: PMC4614192

[20] Outani H, Hamada K, Imura Y, Oshima K, Sotobori T, Demizu Y, Kakunaga S, Joyama S, Imai R, Okimoto T, Naka N, Kudawara I, Ueda T, Araki N, Kamada T, Yoshikawa H. Comparison of clinical and functional outcome between surgical treatment and carbon ion radiotherapy for pelvic chondrosarcoma. Int J Clin Oncol, 2016, 21(1):186-193. https://doi.org/10.1007/s10 147-015-0870-z PMID: 26150259

[21] van Maldegem AM, Gelderblom H, Palmerini E, Dijkstra SD, Gambarotti M, Ruggieri P, Nout RA, van de Sande MA, Ferrari C, Ferrari S, Bovée JVMG, Picci P. Outcome of advanced, unresectable conventional central chondrosarcoma. Cancer, 2014, 120(20):3159-3164. https://doi.org/10.1002/ cncr.28845 PMID: 24995550

[22] Nazeri E, Gouran Savadkoohi M, Majidzadeh-A K, Esmaeili R. Chondrosarcoma: an overview of clinical behavior, molecular mechanisms mediated drug resistance and potential therapeutic targets. Crit Rev Oncol Hematol, 2018, 131:102-109. https:// doi.org/10.1016/j.critrevonc.2018.09.001 PMID: 30293700

[23] Ene R, Cîrstoiu FC, Nica M, Pánti ZA, Panaitescu C, Popescu E, Dumitru AV, Eremia IA Cursaru A, Ene D. Proximal humerus chondrosarcoma in a young male patient. Rom J Morphol Embryol, 2020, 61(3):917-922. https://doi.org/10.47162/RJME. 61.3.31 PMID: 33817734 PMCID: PMC8112793

[24] Thorkildsen J, Taksdal I, Bjerkehagen B, Haugland HK, Børge Johannesen T, Viset T, Norum OJ, Bruland $\varnothing$, Zaikova O. Chondrosarcoma in Norway 1990-2013; an epidemiological and prognostic observational study of a complete national cohort. Acta Oncol, 2019, 58(3):273-282. https://doi.org/10. 1080/0284186X.2018.1554260. Erratum in: Acta Oncol, 2019, 58(3):393. PMID: 30632866

[25] Ranty ML, Michot C, Le Pessot F, Hellot MF, Biga N, Dujardin FH, Simonet J, Billerey C, Metayer J. PAS inclusions, immunoreactive tenascin and proliferative activity in low-grade chondrosarcomas. Pathol Res Pract, 2003, 199(1):29-34. https://doi. org/10.1078/0344-0338-00349 PMID: 12650515

[26] Scully SP, Layfield LJ, Harrelson JM. Prognostic markers in chondrosarcoma: evaluation of cell proliferation and of regulators of the cell cycle. Sarcoma, 1997, 1(2):79-87. https://doi.org/ 10.1080/13577149778344 PMID: 18521206 PMCID: PMC 2395354

[27] Chen YC, Wu PK, Chen CF, Chen WM. Intralesional curettage of central low-grade chondrosarcoma: a midterm follow-up study. J Chin Med Assoc, 2017, 80(3):178-182. https://doi. org/10.1016/j.jcma.2016.10.002 PMID: 27889461 
[28] Dehelean CA, Marcovici I, Soica C, Mioc M, Coricovac D, lurciuc S, Cretu OM, Pinzaru I. Plant-derived anticancer compounds as new perspectives in drug discovery and alternative therapy. Molecules, 2021, 26(4):1109. https://doi. org/10.3390/molecules26041109 PMID: 33669817 PMCID: PMC7922180

[29] Chen X, Yu LJ, Peng HM, Jiang C, Ye CH, Zhu SB, Qian WW. Is intralesional resection suitable for central grade 1 chondrosarcoma: a systematic review and updated meta-analysis. Eur J Surg Oncol, 2017, 43(9):1718-1726. https://doi.org/ 10.1016/j.ejso.2017.05.022 PMID: 28666625

[30] Douis H, Saifuddin A. The imaging of cartilaginous bone tumours. II. Chondrosarcoma. Skeletal Radiol, 2013, 42(5): 611-626. https://doi.org/10.1007/s00256-012-1521-3 PMID: 23053201

[31] Stevenson JD, Laitinen MK, Parry MC, Sumathi V, Grimer RJ, Jeys LM. The role of surgical margins in chondrosarcoma.
Eur J Surg Oncol, 2018, 44(9):1412-1418. https://doi.org/ 10.1016/j.ejso.2018.05.033 PMID: 29929901

[32] Hogendoorn PCW; ESMO/EUROBONET Working Group; Athanasou N, Bielack S, De Alava E, Dei Tos AP, Ferrari S, Gelderblom H, Grimer R, Hall KS, Hassan B, Hogendoorn PCW, Jurgens H, Paulussen M, Rozeman L, Taminiau AHM, Whelan J, Vanel D. Bone sarcomas: ESMO Clinical Practice Guidelines for diagnosis, treatment and follow-up. Ann Oncol, 2010, 21(Suppl 5):v204-v213. https://doi.org/10.1093/annonc/mdq 223 PMID: 20555083

[33] Nota SPFT, Braun Y, Schwab JH, van Dijk CN, Bramer JAM. The identification of prognostic factors and survival statistics of conventional central chondrosarcoma. Sarcoma, 2015, 2015: 623746. https://doi.org/10.1155/2015/623746 PMID: 26633939 PMCID: PMC4655064

\section{Corresponding authors}

loana Delia Horhat, Assistant Professor, MD, PhD, Department of ENT, Victor Babeş University of Medicine and Pharmacy, 6 Revoluţiei 1989 Avenue, 300054 Timişoara, Timiş County, Romania; Phone +40722-281 483, e-mail: deliahorhat@yahoo.com

Alina Andreea Tischer, MD, PhD, Department of ENT, Emergency City Hospital, 6 Revoluţiei 1989 Avenue, 300024 Timişoara, Timiş County, Romania; Phone +40733-106 101, e-mail: flaviabaderca@gmail.com 